\title{
Evaluation of gloves as a water bag coupling agent for therapeutic ultrasound
}

\author{
Lúcio Salustiano de Limaㄹ, Débora Paulino Oliveira² ${ }^{2}$ José Francisco Silva Costa-Júnior ${ }^{1 *}$, \\ Pâmela Alves Pinto ${ }^{1,3}$, Thaís Pionório Omena ${ }^{1,3}$, Rejane Medeiros Costa ${ }^{4}$, Marco Antônio von Krüger ${ }^{1}$, \\ Wagner Coelho de Albuquerque Pereira ${ }^{1}$
}

${ }^{1}$ Biomedical Engineering Program, Federal University of Rio de Janeiro, Rio de Janeiro, RJ, Brazil.

${ }^{2}$ Postgraduate Program on Biotechnology, National Institute of Metrology, Quality and Technology, Rio de Janeiro, RJ, Brazil.

${ }^{3}$ Faculty of Medicine, Federal University of Rio de Janeiro, Rio de Janeiro, RJ, Brazil.

${ }^{4}$ Brazilian National Cancer Institute, Rio de Janeiro, RJ, Brazil.

\begin{abstract}
Introduction: Therapeutic ultrasound (TUS) is a widespread modality in physiotherapy, and the water bag technique is a coupling method employed in the presence of anatomical irregularities in the treatment area. The aim of the present study is to evaluate the acoustic attenuation of the water bag and its effectiveness as a TUS coupling agent. Methods: The rated output powers (ROPs) of the TUS equipment were evaluated based on IEC 61689. Then, a radiation force balance was used to measure ROP with and without a water bag (latex and nitrile gloves filled with deionized water) between a TUS transducer and the cone-shaped target of the balance. Each experiment was performed five times for each nominal power $(0.5,1.0,2.0,3.0,4.0,5.0,6.0$, and $7.0 \mathrm{~W})$ and in the following configurations: without the water bag (A), with nitrile gloves and with (B) and without (C) a height controller, and latex gloves with (D) and without (E) height controller. ROPs obtained in different media were compared. Results: The highest relative error of ROP was $16.72 \%$ for $0.5 \mathrm{~W}$. Although the power values of the equipment were within the range recommended by IEC, there was a significant difference between the ROP values measured with A and with B, C and D. Conclusion: As intensity differences below $0.5 \mathrm{~W} / \mathrm{cm}^{2}$ are considered clinically not relevant, conditions $\mathrm{A}, \mathrm{B}, \mathrm{C}, \mathrm{D}$, or $\mathrm{E}$ can be used interchangeably.
\end{abstract}

Keywords Therapeutic ultrasound, Water bag technique, Acoustic power.

\section{Introduction}

Ultrasound (US) is a mechanical wave with frequency above the human hearing range $(20 \mathrm{~Hz}$ to $20 \mathrm{kHz})$ that transmits energy from one point to another through the vibration of molecules in a medium (Shung, 2006).

Therapeutic ultrasound (TUS) is a technological modality that has been widely used in physical therapy worldwide. One of the therapeutic goals of TUS is to raise the temperature of the treated tissue to the range $40-45^{\circ} \mathrm{C}$

This is an Open Access article distributed under the terms of the Creative Commons Attribution License, which permits unrestricted use, distribution, and reproduction in any medium, provided the original work is properly cited.

How to cite this article: Lima LS, Oliveira DP, Costa-Júnior JFS, Pinto PA, Omena TP, Costa RM, von Krüger MA, Pereira WCA. Evaluation of gloves as a water bag coupling agent for therapeutic ultrasound. Res Biomed Eng. 2017; 33(1):42-49. DOI: 10.1590/24464740.01816 .

*Corresponding author: Biomedical Engineering Program, Federal University of Rio de Janeiro - UFRJ, Avenida Horácio Macedo, 2030, Centro de Tecnologia, Bloco H, CEP 21941-914, Rio de Janeiro, RJ, Brazil. E-mail: jfsc.junior@gmail.com

Received: 15 March 2016 / Accepted: 10 February 2017 to promote pain relief, improve signs of inflammation, increase cell membrane permeability, and enhance the extensibility of collagen.

Moreover, TUS improves blood supply and reduces muscle spasms (Cameron, 2012; Delforge, 2002; Nanda, 2008; Prentice, 2005; Robertson et al., 2006; Watson, 2008a). The literature describes three techniques for the coupling of TUS to the treatment site: the direct-contact method, in which the transducer is coupled to the patient skin using clinical water-based gel; the submersion technique, in which the region receiving US irradiation and the US transducer are separated inside a water tank, with the water acting as the coupling medium; and the water bag method, in which a bag filled with water (or coupling gel) is placed between the skin and the US transducer. The last two techniques have the advantage of being suitable for anatomically uneven surfaces (Cameron, 2012; Kenyon and Kenyon, 2009; Robertson et al., 2006; Starkey, 2013).

In clinical practice, gloves filled with water usually substitute the water bag as a coupling agent because they are easily accessible and have very low cost. 
Although TUS with water glove coupling is a common physiotherapy technique, there is a lack of scientific studies addressing its influence on the desired TUS performance. However, the beneficial physiological effects of TUS are known to depend on several parameters, including the selected dosage and the parameters of the US equipment, such as the US frequency and intensity (Baker et al., 2001; Kollmann et al., 2005). The acoustic energy deposited in a particular area of tissue depends on the calibration of the US equipment, the method of application (direct-contact, submersion and water bag), and the coupling agents (such as different types of hydrogel) between the US transducer and the insonated tissue (Artho et al., 2002; Pye, 1996). Additionally, Klucinec et al. (2000a) quantified the sound energy transmitted through four types of hydrogel (coupling medium) manufactured by different companies and observed a considerable difference in the transmissivity of the US wave depending on the used hydrogel, with the transmitted voltage ranging between $42.8 \pm 5.9 \%$ and $77.2 \pm 4.6 \%$. To maximize the energy transfer from an ultrasonic wave emitted by a transducer to the irradiated biological tissue, the acoustic impedance of the coupling medium must optimize the matching between the acoustic impedances of the transducer and the irradiated medium. When no coupling medium is used, the largest portion of the acoustic energy can be reflected on the transducer-air interface owing to the large impedance mismatch between these mediums. Nevertheless, Poltawski and Watson (2007) reported that depending on the composition, the absorption and scattering coefficients of the coupling medium may induce a loss of energy of the acoustic beam that reaches the tissue. Besides, the impedance of the coupling medium itself can reduce the wave energy transmitted to the tissue. Another study reported that the transmitted power is affected by the temperature of the coupling agents (Oshikoya et al., 2000) because of changes in the coupling medium density, which is related to the acoustic impedance. Guirro et al. (2001) evaluated the transmissivity of a balloon and a latex glove filled with hydro-soluble gel to be $2 \%$ and $18 \%$, respectively, using degassed water as a reference.

Klucinec et al. (2000b) stated that the literature on the transmissivity of gel pads, bladder techniques, and water bath immersion is inconclusive and these topics require further investigation. Therefore, this study aims to evaluate the efficacy of TUS with the water bag technique by comparing the measurement of US power with and without the use of water-filled latex and nitrile gloves, which is an arrangement commonly used by physical therapy professionals.

\section{Methods}

The following equipment was used in the experiments: a radiation force balance (UPM-DT 1AV; Ohmic Instruments Co., St. Charles, Mo, USA) with a display resolution of $\pm 2 \mathrm{~mW}$, one pair of latex gloves size $\mathrm{M}$ (Lagrotta Azzurra, São Paulo, SP, Brazil), one pair of nitrile gloves size M (Lagrotta Azzurra, São Paulo, SP, Brazil), ultrapure and degassed water, and a physical therapy US device with a circular-plane piston transducer (Seven Pro 977; Quark Medical Products, Piracicaba, SP, Brazil). According to the manufacturer of this device, the effective radiating area (ERA) of the transducer was $3.8 \mathrm{~cm}^{2}$, the beam non-uniformity ratio was lower than 6 , and the ceramic with $2.48 \mathrm{~cm}$ in diameter. The thickness of the latex and nitrile gloves was $12 \mu \mathrm{m}$. The experimental setup is illustrated in Figure 1A.

The radiation force balance was composed of a $45^{\circ}$ air-backed cone target immersed in a tank with rubber-coated inner surface. A positioning clamp was employed to keep the transducer immersed in ultrapure and degassed water above the conical target. As the physical therapy US device was configured to operate in continuous mode, the ultrasonic transducer generated sinusoidal continuous waves with $1 \mathrm{MHz}$ frequency. This wave propagated through the water and was reflected from the conical target, exerting an ultrasonic force on this target. The vertical component of this force was registered as an increase in the apparent mass of the target. This measurement was performed by the support assembly that connected the target to an electronic digital balance. Using this approach, an apparent mass of $1 \mathrm{~g}$ corresponded to a power of $14.65 \mathrm{~W}$. After being reflected by the target, the ultrasonic energy was absorbed by the rubber lining of the tank.
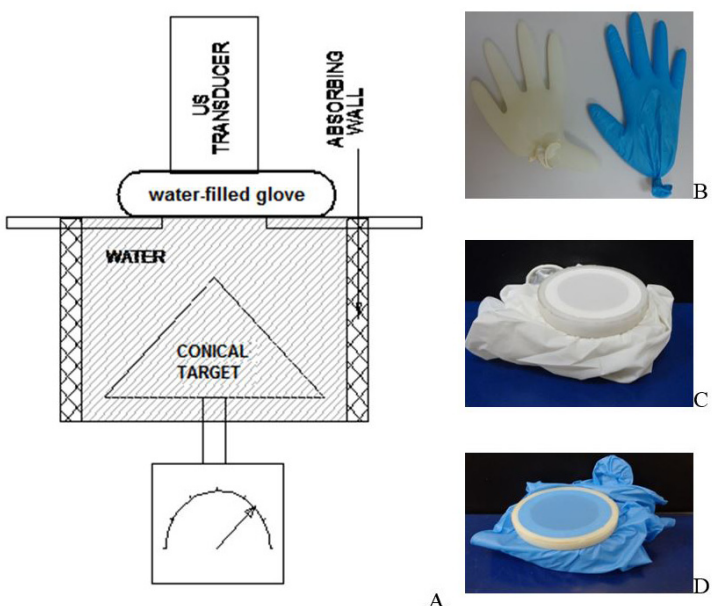

Figure 1. (A) Experimental setup; (B) Latex and nitrile gloves filled with water. PVC ring filled with water and covered with (C) latex and (D) nitrile gloves. 
After preparing the experimental setup as shown in Figure 1A, the ultrasonic equipment remained switched off for 5 min before starting the experiment, as this is the time recommended by the manufacturer for the radiation force balance to stabilize. Then, a body mass of $1 \mathrm{~g}$, which was provided by the manufacturer, was employed to calibrate the equipment.

Ultrapure and degassed water was used in the experiments because in common water, the concentration of diluted gases can promote different degrees of US cavitation, which can influence the US therapy performance (Wells, 1977). Purified water was obtained from an Elga Purelab Option Q purification system (Elga Labwater, High Wycombe, Bucks, UK). To minimize cavitation, a vacuum chamber (model 166; Primar Equipment and Services Ltd., Itu, SP, Brazil) was used to remove diluted gases from the water, as recommended by International Electrotechnical Commission (IEC) 61689 (International..., 2007). The water temperature was $25.0 \pm 0.4{ }^{\circ} \mathrm{C}$.

\section{Experimental procedure}

The first experimental setup, without the gloves, was used to determine the power emitted by the TUS equipment for all the applied nominal power values (calibration curve). The measurement served as a reference for the power measured when gloves were introduced in the experiments.

The second experimental setup included a glove filled with deionized water to evaluate its influence on the measured power. This condition simulates the water bag technique.

In an effort to perform measurements with a more controlled glove thickness, the measurement was repeated with the same TUS power, this time using a PVC cylinder ( $12.8 \mathrm{~mm}$ in height, $32.65 \mathrm{~mm}$ internal and $44.00 \mathrm{~mm}$ external diameters) filled with deionized water and covered with two gloves. A PVC ring was used to ensure that there were no wrinkles on the glove surface and that the distance between the two glove membranes corresponded to the height of the ring. The PVC ring alone, filled with water, and covered with the latex and nitrile gloves are shown in Figure 1B-D.

Each experiment was repeated five times at each nominal power of the equipment $(0.5,1.0,2.0,3.0,4.0,5.0$, 6.0 , and $7.0 \mathrm{~W}$ ) without the gloves (reference) and with the two gloves of different materials (latex and nitrile), with and without the height controller (PVC cylinder). All experiments were performed with the same equipment at the same time of day (afternoon) and by one operator, at an ambient temperature of $25 \pm 2{ }^{\circ} \mathrm{C}$. Attention was paid to prevent the formation of air bubbles inside the glove when closing it to avoid the decrease of the output power.

\section{Evaluation of output power of the TUS equipment}

Using the reference experimental setup (without gloves), the output power was verified to be within the range recommended by IEC 61689 (2007) for each nominal value $( \pm 20 \%)$. The error between the measured and the nominal power (expressed as percentage) was calculated using Equation 1:

$$
\text { Error }=\left|\left(\frac{P_{\text {Measured }}-P_{\text {Nominal }}}{P_{\text {Nominal }}}\right) \cdot 100\right|
$$

\section{Statistical analysis}

Statistical analyses were performed with the software BioEstat 5.0 (Ayres et al., 2007). The Shapiro-Wilk test was used to analyze the normality of the data from the measured power values for each experimental condition. The next step was to examine the ratio between the highest and the lowest standard deviations of the measured power for all nominal values and experimental conditions. If the data do not have a normal distribution and/or the ratio obtained was greater than 2 , a nonparametric test (Kruskal-Wallis test) was performed to verify if the output power variations for the different experimental conditions were statistically significant (Moore, 2006). When the Kruskal-Wallis test results exhibited significant differences, the Student-Newman-Keuls multiple comparisons post-test was performed. All results were considered statistically significant if $\mathrm{p}$-value $<0.05$.

\section{Results}

The mean and standard deviation of the output power were determined for the five different experimental conditions, which were categorized as follows: A: deionized water without the presence of gloves; B, C: nitrile gloves filled with deionized water with (B) and without (C) height control by the PVC ring; D, E: latex gloves filled with deionized water with (D) and without (E) height control by the PCV ring. The results are shown in Table 1.

Table 2 shows the relative error (\%) of rated output power estimated in condition A with reference to the nominal power.

The Shapiro-Wilk test was applied to the 40 distribution groups $(5$ techniques and 8 acoustic power values) of the measured power and determined that four of them did not have a normal distribution ( 0.5 and $3.0 \mathrm{~W}$ for configuration $\mathrm{A}$ and 2.0 and $5.0 \mathrm{~W}$ for D). Additionally, the ratio between the highest and lowest standard deviations was evaluated to be higher than 2 in four cases (for 1.0, 4.0, 6.0, and 7.0 W). Then, the Kruskal-Wallis test was used to compare the power measurements obtained for all conditions and nominal 
Table 1. Nominal power, mean, and standard deviation of the measured power for A (deionized water without glove), B (nitrile glove filled with deionized water and height control by a PCV ring), C (nitrile glove filled with deionized water), D (latex glove filled with deionized water and height control by a PCV ring), and E (latex glove filled with deionized water).

\begin{tabular}{cccccc}
\hline \multirow{2}{*}{ Nominal power [W] } & \multicolumn{5}{c}{ Measured power [W] } \\
\cline { 2 - 6 } & $\mathbf{A}$ & $\mathbf{B}$ & $\mathbf{C}$ & $\mathbf{D}$ & $\mathbf{E}$ \\
\hline 0.5 & $0.42 \pm 0.03$ & $0.41 \pm 0.02$ & $0.42 \pm 0.02$ & $0.43 \pm 0.03$ & $0.46 \pm 0.02$ \\
1.0 & $0.94 \pm 0.02$ & $0.81 \pm 0.05$ & $0.85 \pm 0.04$ & $0.87 \pm 0.02$ & $0.92 \pm 0.01$ \\
2.0 & $1.85 \pm 0.03$ & $1.81 \pm 0.03$ & $1.69 \pm 0.02$ & $1.73 \pm 0.00$ & $1.81 \pm 0.01$ \\
3.0 & $2.89 \pm 0.04$ & $2.66 \pm 0.01$ & $2.67 \pm 0.01$ & $2.73 \pm 0.05$ & $2.86 \pm 0.02$ \\
4.0 & $4.03 \pm 0.04$ & $3.55 \pm 0.02$ & $3.72 \pm 0.01$ & $3.80 \pm 0.04$ & $3.99 \pm 0.02$ \\
5.0 & $5.17 \pm 0.05$ & $4.57 \pm 0.08$ & $4.70 \pm 0.01$ & $4.87 \pm 0.04$ & $5.05 \pm 0.01$ \\
6.0 & $6.27 \pm 0.03$ & $5.58 \pm 0.05$ & $5.59 \pm 0.01$ & $5.73 \pm 0.10$ & $6.09 \pm 0.02$ \\
7.0 & $7.14 \pm 0.04$ & $6.36 \pm 0.04$ & $6.40 \pm 0.03$ & $6.61 \pm 0.07$ & $6.97 \pm 0.02$ \\
\hline
\end{tabular}

Table 2. The nominal power $(\mathrm{W})$ and the relative error $(\%)$ of measured power for A (deionized water without glove).

\begin{tabular}{cc}
\hline Nominal power $(\mathbf{W})$ & Error $(\%)$ \\
\hline 0.5 & 16.72 \\
1.0 & 6.12 \\
2.0 & 7.42 \\
3.0 & 3.72 \\
4.0 & 0.79 \\
5.0 & 3.31 \\
6.0 & 4.58 \\
7.0 & 2.04 \\
\hline
\end{tabular}

powers (the null hypothesis of this test is that the means of the A, B, C, D, and E groups are the same). All p-value results calculated using the Kruskal-Wallis test were below 0.01 . Therefore, the multiple-comparison Student-Newman-Keuls test was applied. The difference between the average measured power and the results of the multiple-comparison test are presented in Table 3. The superscript $\checkmark$ indicates that $p$-value $<0.05$ for the compared pair of conditions.

\section{Discussion}

Ultrasonic transmission from the transducer to the skin depends on the presence of a coupling medium between the transducer surface and the tissue. When the tissue surface is irregular, the transducer contact may be compromised.

On the other hand, a coupling medium with a high absorption coefficient, with air bubbles, or agents that alter the energy transmission is not recommended. Furthermore, the presence of a coupling agent facilitates the matching of the acoustic impedances of the tissue and transducer (Watson, 2008b).

The water bag technique is an indirect-contact and low-cost US coupling approach. Its use is very common in everyday clinical practice, especially for the treatment of anatomically uneven surfaces (Guirro and Guirro, 2002; Guirro et al., 2001; Starkey, 2013). However, scientific studies investigating this technique are scarce (Guirro et al., 2001; Klucinec et al., 2000b).

The study conducted by Poltawski and Watson (2007) on the performance of different coupling agents (gels) on US transmission showed differences in the energy transmission of ultrasonic waves that had no clinical significance.

Klucinec et al. (2000b) demonstrated a reduction in the acoustic energy transfer when degassed and tap water in latex gloves, gel in a latex glove, and a gel-filled condom were used as coupling media. The same effect was observed in this work for conditions B, C, D, and E for all nominal powers except $0.5 \mathrm{~W}$ (see Table 1). Generally, the measured powers in the control configuration (A) did not show significant differences from those obtained in E, with the exception of the nominal power of $0.5 \mathrm{~W}$. The mean difference between group pairs exhibited a general tendency to increase as the nominal power increased.

Guirro et al. (2001) indicated that the coupling means (balloon and latex glove filled with hydro-soluble gel) employed in their experiments presented a high attenuation coefficient, i.e., had low transmissivity. Nevertheless, the present study shows (Table 1) that the highest difference between the mean power values of $\mathrm{A}$ and those measured in configurations containing a glove $(\mathrm{B}, \mathrm{C}, \mathrm{D}$, or $\mathrm{E})$ was below $15.0 \%$, irrespective of the nominal power. In addition, the smallest mean power difference was obtained between $\mathrm{A}$ and $\mathrm{E}(<3.0 \%)$, regardless of the nominal power.

\section{Comparison of conditions $A, B$ and $C$}

The significant difference between the power values measured at different experimental conditions (A-B and/or A-C) that was observed in some situations (nominal power 4-7 W) is noteworthy because, in some cases, the averages of the compared pairs differed by 
Table 3. Difference between the average measured power and the Student-Newman-Keuls post hoc test results for all conditions (A, B, C, D, and E). The superscript $\sqrt{ }$ indicates that $\mathrm{p}<0.05$

\begin{tabular}{|c|c|c|c|c|c|}
\hline Nominal Power (W) & $\mathbf{A}$ & B & C & D & $\mathbf{E}$ \\
\hline \multirow{4}{*}{0.5} & $\mathrm{~A}$ & 0.00 & 0.00 & 0.02 & $0.04^{\checkmark}$ \\
\hline & $\mathrm{B}$ & & 0.01 & 0.02 & $0.05^{\checkmark}$ \\
\hline & $\mathrm{C}$ & & & 0.01 & $0.04^{\checkmark}$ \\
\hline & $\mathrm{D}$ & & & & 0.03 \\
\hline \multirow{4}{*}{1.0} & A & $0.13^{\checkmark}$ & $0.09^{\checkmark}$ & $0.07^{\checkmark}$ & 0.02 \\
\hline & $\mathrm{B}$ & & 0.04 & 0.06 & $0.11^{\checkmark}$ \\
\hline & $\mathrm{C}$ & & & 0.02 & $0.07^{\checkmark}$ \\
\hline & $\mathrm{D}$ & & & & 0.05 \\
\hline \multirow{4}{*}{2.0} & A & 0.04 & $0.16^{\checkmark}$ & $0.13^{\checkmark}$ & 0.04 \\
\hline & $\mathrm{B}$ & & $0.11^{\checkmark}$ & 0.08 & 0.00 \\
\hline & $\mathrm{C}$ & & & 0.03 & $0.12^{\checkmark}$ \\
\hline & $\mathrm{D}$ & & & & 0.08 \\
\hline \multirow{4}{*}{3.0} & $\mathrm{~A}$ & $0.23^{\checkmark}$ & $0.22^{\checkmark}$ & $0.16^{\checkmark}$ & 0.03 \\
\hline & $\mathrm{B}$ & & 0.02 & 0.08 & $0.20^{\checkmark}$ \\
\hline & $\mathrm{C}$ & & & 0.06 & $0.18^{\checkmark}$ \\
\hline & $\mathrm{D}$ & & & & 0.12 \\
\hline \multirow{4}{*}{4.0} & $\mathrm{~A}$ & $0.48^{\checkmark}$ & $0.32^{\checkmark}$ & $0.23^{\checkmark}$ & 0.04 \\
\hline & $\mathrm{B}$ & & 0.16 & $0.25^{\checkmark}$ & $0.44^{\checkmark}$ \\
\hline & $\mathrm{C}$ & & & 0.08 & $0.28^{\checkmark}$ \\
\hline & $\mathrm{D}$ & & & & 0.19 \\
\hline \multirow{4}{*}{5.0} & A & $0.59^{\checkmark}$ & $0.47^{\checkmark}$ & $0.30^{\checkmark}$ & 0.11 \\
\hline & $\mathrm{B}$ & & 0.12 & $0.29^{\checkmark}$ & $0.48^{\checkmark}$ \\
\hline & $\mathrm{C}$ & & & 0.17 & $0.36^{\checkmark}$ \\
\hline & $\mathrm{D}$ & & & & 0.19 \\
\hline \multirow{4}{*}{6.0} & A & $0.70^{\checkmark}$ & $0.68^{\checkmark}$ & $0.55^{\checkmark}$ & 0.19 \\
\hline & B & & 0.02 & 0.15 & $0.51^{\checkmark}$ \\
\hline & $\mathrm{C}$ & & & 0.13 & $0.50^{\checkmark}$ \\
\hline & $\mathrm{D}$ & & & & 0.36 \\
\hline \multirow{4}{*}{7.0} & $\mathrm{~A}$ & $0.78^{\checkmark}$ & $0.74^{\checkmark}$ & $0.53^{\checkmark}$ & 0.18 \\
\hline & B & & 0.04 & 0.25 & $0.61^{\checkmark}$ \\
\hline & $\mathrm{C}$ & & & 0.21 & $0.57^{\checkmark}$ \\
\hline & D & & & & 0.35 \\
\hline
\end{tabular}

more than $0.45 \mathrm{~W}$. This was observed for the nominal powers $4.0 \mathrm{~W}$ (A-B), 5.0 W (A-B and A-C), $6.0 \mathrm{~W}$ (A-B and $\mathrm{A}-\mathrm{C}$ ), and 7.0 W (A-B and $\mathrm{A}-\mathrm{C}$ ). When comparing the measurements obtained in $\mathrm{B}$ and $\mathrm{C}$ configurations, the highest power difference was $0.16 \mathrm{~W}$ for a nominal power of $4 \mathrm{~W}$, which is not clinically relevant (Itakura et al., 2012).

In summary, the experimental results exhibited no clinical difference for the nitrile gloves with or without controlled height.

\section{Comparison of conditions $A, D$ and $E$}

The results of the pairwise comparison test showed that only the output power measured with the experimental condition pair A-E was not significantly different (except for the nominal power of $0.5 \mathrm{~W}$ ). Nevertheless, the difference between the averages was below $0.19 \mathrm{~W}$, which is clinically irrelevant. The corresponding difference for the pair A-D was higher than $0.5 \mathrm{~W}$ for nominal powers of 6 and $7 \mathrm{~W}$. When comparing the measurements obtained in $\mathrm{D}$ and $\mathrm{E}$, the difference between the averages reached $0.36 \mathrm{~W}$ for nominal power $6 \mathrm{~W}$, which is not clinically relevant. In other words, for latex gloves, there no clinically relevant difference was observed between the measured powers, with or without height control.

\section{Comparison of conditions $B, C, D$ and $E$}

No significant difference was observed for the experimental conditions B and D (except for nominal powers 4.0 and $5.0 \mathrm{~W}$ ). Nevertheless, the mean power differences were not clinically relevant. On the other hand, significant difference was observed for the experimental conditions $\mathrm{C}$ and $\mathrm{E}$ for power values 6 and $7 \mathrm{~W}$. 


\section{General comments}

The reduction of the output power and the differences observed in the study can be interpreted by phenomena such as cavitation and standing waves and by considering the intrinsic factors of the coupling media, such as the thickness of the coupling agent layer, the positioning of the glove membrane, the target size, and beam divergence (Casarotto et al., 2004; Poltawski and Watson, 2007; Shaw and Hodnett, 2008; Wells, 1977).

Moreover, in the configuration containing a glove filled with deionized water and height control by the $\mathrm{PVC}$ ring (B and $\mathrm{D})$, reverberation can occur in the presence of two parallel membranes, causing destructive interference of the wave, which can reduce the power of the wave in the region of interest.

Another factor that can reduce US power is the distance between the treatment region and the TUS transducer. Therefore, a power measurement was conducted to compare a glove filled with deionized water without controlled height $(\mathrm{h} \approx 31.0 \mathrm{~mm})$ and one with controlled height $(\mathrm{h}=12.8 \mathrm{~mm})$. However, the mentioned factor has not been proven by experiments, for the same kind of gloves. Only in one case (B-C for nominal power of $2.0 \mathrm{~W}$ ) the average of rated output power was significantly different. Nevertheless, the difference between the averages of D-E is greater than that of B-C. According to recommendations (Low and Reed, 2000; Wells, 1977), all experiments were performed with the $45^{\circ}$ air-backed cone target positioned in the near field $\left(a^{2} / \lambda=1.24^{2} / 0.15 \cong 10.3 \mathrm{~cm}\right)$ because the treatment area is recommended to be placed in this region.

Although the relative error of the rated output power is within the range recommended by IEC 61689 (International..., 2007), the observed variation of the measured acoustic power can be also attributed to the bath immersion technique. Klucinec et al. (2000b) showed that transmissivity is reduced when this technique is employed.

Similarly to latex, nitrile is a type of rubber. Because of the different chemical compositions of these materials, their attenuation coefficients also differ. For this reason, the rated output powers measured using latex and nitrile gloves have statistically different values.

As household water is known to contain several diluted gases, which facilitates the occurrence of cavitation (Kenyon and Kenyon, 2009), in this study, only deionized and degassed water was used to minimize this phenomenon. Deionized water was placed in the vacuum chamber to extract the diluted gases and thus avoid the formation of bubbles inside the glove. Although this does not realistically reflect the practices followed in clinics, it serves as a reference. If air bubbles form in deionized water, then, the use of tap water would induce cavitation. To exemplify this phenomenon, a glove was filled with tap water and irradiated with a TUS frequency of $1 \mathrm{MHz}$ and power of $7 \mathrm{~W}$ for $5 \mathrm{~min}$ using a clinical gel as the coupling medium between the transducer and the glove. Even small cavitation can form microbubbles, which can assemble to create a bubble (see Figure 2). Microbubbles (wavelength lower than $1.5 \mathrm{~mm}$ ) generate scattering of the US wave, and bubbles induce reflection of the incident wave. In both cases, there is a reduction in the power of the transmitted wave.

In the experimental condition shown in Figure 2, the bubble formation caused severe attenuation, leading to acoustic power measurements below the nominal power on several occasions.

Consequently, as deionized and degassed water are not commonly available in clinics, the use of the water bag technique in clinical practices can lead to ineffective therapy. When tap water is employed, cavitation occurs to the point of clear bubble formation, considerably decreasing the measured power to values below the detection limit of the radiation force balance. Otherwise, since the transducer does not stay still, but the physician moves it around the body region, there must exist another biophysical effect, which needs future research. A possible explanation may be that ultrasonic beam will not be totally reflected during the irradiation.

An interesting effect that has not been sufficiently studied is the increase in the average power difference of the compared techniques with increasing nominal power, which was observed in the values of Table 3 .

The highest difference between the rated output powers $(0.78 \mathrm{~W})$ was obtained for the pair A-B and nominal power $7 \mathrm{~W}$. From the clinical point view, the important parameter is intensity (power/ERA). Therefore, we can estimate the intensity by using the nominal value of the ERA of the ultrasonic transducer, $3.8 \mathrm{~cm}^{2}$, and the

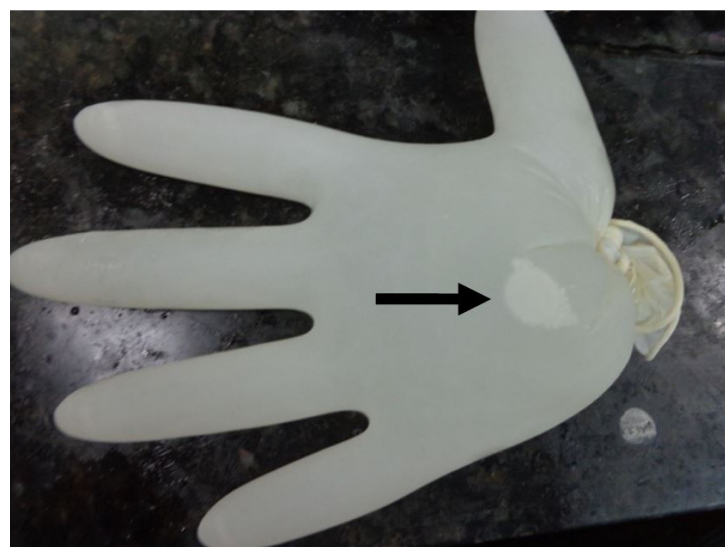

Figure 2. Formation of air bubble cluster, indicated by the arrow, on a latex glove filled with common tap water. The coupling gel was removed for a better view of the bubbles. 
highest difference between the intensities is $0.21 \mathrm{~W} / \mathrm{cm}^{2}$ $\left(0.78 \mathrm{~W} / 3.8 \mathrm{~cm}^{2}\right)$. In our study, the ERA was not measured; as it is usually smaller than the nominal ERA, we assumed the extreme case in which the real ERA was 50\% lower than the nominal one. In this case, the highest difference between the intensities was $0.41 \mathrm{~W} / \mathrm{cm}^{2}\left(0.78 \mathrm{~W} / 1.9 \mathrm{~cm}^{2}\right)$, which is lower than $0.5 \mathrm{~W} / \mathrm{cm}^{2}$. From the clinical point of view, intensity differences below $0.5 \mathrm{~W} / \mathrm{cm}^{2}$ are not considered relevant; thus, conditions $\mathrm{A}, \mathrm{B}, \mathrm{C}, \mathrm{D}$, and $\mathrm{E}$ are equivalent.

In summary, the experimental results showed that the water bag technique with a latex glove filled with deionized and degassed water (E) can be used as a coupling agent. This is because the statistical results indicated that the power values measured with this technique and the control (A) were not statistically significant and the difference between the averages of the power for these methods was below $0.19 \mathrm{~W}$. In addition, this technique is a viable option to treat irregular anatomic regions, because deionized and degassed water is used in the process. The use of common tap water can increase cavitation and US attenuation, which can compromise the success of the therapy. Additionally, it is advisable to avoid the formation of air bubbles inside the glove when closing it, as this could cause greater attenuation of the ultrasonic wave beam, reducing the effective power and treatment effectiveness. On the other hand, as intensity differences lower than $0.5 \mathrm{~W} / \mathrm{cm}^{2}$ are considered clinically irrelevant, conditions A, B, C, D, and E can be used interchangeably.

Oshikoya et al. (2000) observed that the temperature of the coupling medium may reduce the measured power, which may influence the treatment effectiveness. However, throughout the present experiment, both the ambient and the water temperature were $25.0 \pm 0.4{ }^{\circ} \mathrm{C}$ and the measured power could not be affected by the temperature of the coupling medium.

Further studies must be performed on this topic employing TUS equipment of different manufacturers.

\section{Acknowledgements}

The authors thank FAPERJ, CNPq, and CAPES for financial support.

\section{References}

Artho PA, Thyne JG, Warring BP, Willis CD, Brismée JM, Latman NS. A calibration study of therapeutic ultrasound units. Physical Therapy. 2002; 82(3):257-63. PMid:11869154.

Ayres M, Ayres Júnior M, Ayres DL, Santos AS. BioEstat 5.0: aplicações estatísticas nas áreas das ciências biológicas e médicas. 5th ed. Belém: Sociedade Civil Mamirauá/CNPq; 2007.

Baker KG, Robertson VJ, Duck FA. A review of therapeutic ultrasound: biophysical effects. Physical Therapy. 2001; 81(7):1351-8. PMid:11444998.
Cameron MH. Physical agents in rehabilitation: from research to practice. 4th ed. St. Louis: Saunders Elsevier; 2012.

Casarotto RA, Adamowski JC, Fallopa F, Bacanelli F. Coupling agents in therapeutic ultrasound: acoustic and thermal behavior. Archives of Physical Medicine and Rehabilitation. 2004; 85(1):162-5. PMid:14970985. http://dx.doi.org/10.1016/ S0003-9993(03)00293-4.

Delforge G. Musculoskeletal trauma: implications for sports injury management. Champaign: Human Kinetics; 2002.

Guirro E, Guirro R. Fisioterapia dermato-funcional: fundamentos, recursos e patologias. 3rd ed. São Paulo: Manole; 2002.

Guirro R, Cancelieri AS, Sant'Anna IL. Avaliação dos meios intermediários utilizados na aplicação do ultra-som terapêutico. Rev Bras Fisiot. 2001; 5(2):49-52.

International Electrotechnical Commission. IEC 61689:2007. Ultrasonics: physiotherapy systems: field specifications and methods of measurement in the frequency range $0.5 \mathrm{MHz}$ to $5 \mathrm{MHz}$. Geneva: IEC; 2007.

Itakura DA, Magas V, Neves EB, Nohama P. Alteração da temperatura nos tecidos biológicos com a aplicação do ultrassom terapêutico: uma revisão. Fisioterapia em Movimento. 2012; 25(4):857-68. http://dx.doi.org/10.1590/ S0103-51502012000400019.

Kenyon K, Kenyon J. The physiotherapist's pocketbook: essential facts at your fingertips. 2nd ed. London: Churchill Livingstone Elsevier; 2009.

Klucinec B, Scheidler M, Denegar C, Domholdt E, Burgess $\mathrm{S}$. Effectiveness of wound care products in the transmission of acoustic energy. Physical Therapy. 2000a; 80(5):469-76. PMid:10792857.

Klucinec B, Scheidler M, Denegar C, Domholdt E, Burgess S. Transmissivity of coupling agents used to deliver ultrasound through indirect methods. The Journal of Orthopaedic and Sports Physical Therapy. 2000b; 30(5):263-6. PMid:10817413. http://dx.doi.org/10.2519/jospt.2000.30.5.263.

Kollmann C, Vacariu G, Schuhfried O, Fialka-Moser V, Bergmann H. Variations in the output power and surface heating effects of transducers in therapeutic ultrasound. Archives of Physical Medicine and Rehabilitation. 2005; 86(7):1318-24. PMid:16003657. http://dx.doi.org/10.1016/j.apmr.2005.02.001.

Low J, Reed A. Electrotherapy explained: principles and practice. 3rd ed. Oxford: Butterworth Heinemann; 2000.

Moore DS. The basic practice of statistics. 4th ed. New York: W. H. Freeman \& Co LTD; 2006.

Nanda BK. Electrotherapy simplified. New Delhi: Jaypee Brothers Medical; 2008.

Oshikoya CA, Shultz SJ, Mistry D, Perrin DH, Arnold BL, Gansneder BM. Effect of coupling medium temperature on rate of intramuscular temperature rise using continuous ultrasound. Journal of Athletic Training. 2000; 35(4):417-21. PMid:16558655.

Poltawski L, Watson T. Relative transmissivity of ultrasound coupling agents commonly used by therapists in the UK. Ultrasound in Medicine \& Biology. 2007; 33(1):120-8. PMid:17189054. http://dx.doi.org/10.1016/j.ultrasmedbio.2006.07.026. 
Prentice WE. Therapeutic modalities in rehabilitation. 3rd ed. Nova York: McGraw-Hill Medical; 2005.

Pye S. Ultrasound therapy equipment: does it perform? Physiotherapy. 1996; 82(1):39-44. http://dx.doi.org/10.1016/ S0031-9406(05)66996-9.

Robertson V, Ward A, Low J, Reed A. Electrotherapy explained: principles and practice. 4 th ed. Oxford: Butterworth-Heinemann Elsevier; 2006.

Shaw A, Hodnett M. Calibration and measurement issue for therapeutic ultrasound. Ultrasonics. 2008; 48(4):234-52. PMid:18234261. http://dx.doi.org/10.1016/j.ultras.2007.10.010.
Shung KK. Diagnostic ultrasound: imaging and blood flow measurements. Boca Raton: CRC Press; 2006.

Starkey C. Therapeutic modalities. 4th ed. Philadelphia: F.A. Davis; 2013.

Watson T. Electrotherapy: evidence-based practice. 12th ed. Philadelphia: Churchill Livingstone Elsevier; 2008a.

Watson T. Ultrasound in contemporary physiotherapy practice. Ultrasonics. 2008b; 48(4):321-9. PMid:18466945. http://dx.doi. org/10.1016/j.ultras.2008.02.004.

Wells PNT. Biomedical ultrasonics. London: Academic Press; 1977. 